\title{
APLICAÇÃO DE BIM EM INFRAESTRUTURAS DE TRANSPORTES
}

\author{
Maria João Falcão Silva ${ }^{(1)}$, Paula Couto ${ }^{(1)}$, Simona Fontul ${ }^{(1)}$ \\ (1) Laboratório Nacional de Engenharia Civil, Lisboa
}

\begin{abstract}
Resumo
As infraestruturas de transportes, como estradas, ferrovias e aeroportos, encontram-se na atualidade sujeitas a um aumento cada vez maior do tráfego, devendo a sua reabilitação, caso necessário, ser realizada de forma eficiente. Nesse processo, a monitorização da capacidade estrutural e funcional das infraestruturas de transportes usando ensaios não destrutivos é realizada de forma sistemática. É importante aproveitar essas medidas para planear intervenções de reabilitação futura.

O BIM, por ser uma metodologia relativamente recente e inovadora, afigura-se como uma ferramenta da maior importância, e com grande potencial quando aplicada ao estudo de infraestruturas de transporte novas e/ou a reabilitar.

Neste artigo, são apresentados quatro casos-estudo da aplicação de BIM na reabilitação de infraestruturas de transportes existentes. No caso de pavimentos de estradas e aeroportos, foi estudada a integração da componente estrutural na modelação. Em relação às ferrovias, foi abordada a construção de uma zona de transição, bem como seu comportamento durante os três primeiros anos após a entrada em serviço. Dessa forma, é possível obter um modelo que contenha informações sobre a condição estrutural resultante de inspeções contínuas efetuadas. As principais conclusões obtidas são sumariamente apresentadas, sendo identificadas as vantagens da abordagem considerada.
\end{abstract}

\section{Introdução}

O Building Information Modelling (BIM) é uma metodologia adequada para todo o setor da arquitetura, engenharia, construção e operação (AECO), que permite a representação das características estruturais e funcionais de uma construção, incluindo atividades associadas, entre outras informações. A principal característica do BIM é o sistema de modelação tridimensional que inclui a gestão, a partilha e a troca de dados ao longo do ciclo de vida de uma estrutura, onde cada elemento ou objeto tem associadas informações de seus dados físicos. A metodologia BIM permite uma melhor coordenação e colaboração entre os intervenientes do 
projeto, permitindo acesso simultâneo e em tempo real, uma rápida deteção de conflitos entre componentes e, consequentemente, uma redução de custos associados. No que diz respeito à avaliação de estradas, aeroportos e infraestruturas ferroviárias procura-se, com recurso à metodologia BIM, identificar a informação referente às contribuições de ensaios não destrutivos efetuados sobre as infraestruturas estudadas, para o controle de qualidade durante a construção e realizar testes de carga durante a sua vida em serviço. Os ensaios de carga não destrutivos permitem a obtenção de informação para incorporar em modelos de comportamento estrutural. Exemplos de possíveis aplicações da metodologia BIM à monitorização e reabilitação de pavimentos e ferrovias são apresentados no presente artigo.

\section{Building Information Modelling (BIM)}

BIM é um termo que tem vindo a ser introduzido gradualmente na indústria da construção nas últimas décadas e é um dos desenvolvimentos promissores no setor AECO. Trata-se de uma metodologia que mostra mudanças muito significativas em relação à abordagem atual de projeto, construção e manutenção de infraestruturas. Para resolver alguns dos problemas do setor, o BIM leva ao uso de métodos coerentes de informações do projeto em relação à centralização de computadores. A tecnologia BIM compreende um conjunto de dados, relacionados a elementos paramétricos, a base do processo de modelação. Um elemento paramétrico é um objeto digital que contém, além dos parâmetros geométricos, várias características específicas das propriedades materiais e físicas [1].

A especificação de nível de desenvolvimento (LOD) foi desenvolvida é uma referência que permite aos profissionais da indústria da AECO especificar e articular com um alto nível de clareza de conteúdo e confiabilidade dos modelos 3D em vários estágios. A especificação LOD adiciona contexto em relação à alta transparência e confiabilidade dos modelos BIM, como resultado de várias fases da construção. Também define características dos elementos que compõem o modelo em diferentes especialidades de construção, a fim de esclarecer os profissionais sobre possíveis limitações dos modelos que foram recebidos. No entanto, deve-se notar que o LOD não substitui um plano de implementação do projeto no BIM, mas deve ser usado como um plano [2].

$\mathrm{Na}$ implementação do BIM, o setor da AECO é incentivado a apresentar uma maior transparência e colaboração entre fornecedores, aumentando positivamente a reputação do setor. Vários países têm vindo a adotar essa metodologia nos últimos anos, à medida que as questões de tecnologia e implementação melhoram e mudanças significativas são observadas com a implementação e o uso do BIM [3].

\section{BIM na gestão de pavimentos}

Pretende-se avaliar a aplicabilidade prática do conceito de BIM implementado no caso de estudo, que busca enfatizar a definição de elementos e a representação de informações por meio de metodologias mais rápidas e maior natureza organizacional. Isso resulta na otimização da infraestrutura, aumentando os níveis de segurança, qualidade e custo. Nesse sentido, é mais importante entender, a priori, as metodologias de inspeção e monitorização de infraestruturas existentes e entender como os dados podem ser estruturados para o estabelecimento de um 
modelo de registo das informações em bases de dados estruturadas. por objetos. A primeira fase do trabalho é baseada nos principais aspetos das campanhas de avaliação de pavimentos rodoviários, implementadas em um estudo de caso de um pavimento rodoviário em serviço [4], tendo sido concebido em ambiente BIM, onde é apresentado o potencial dessa ferramenta na monitorização da condição dos pavimentos. Os dados resultantes das campanhas de avaliação são monitorizados, visando entender a evolução dos diferentes componentes dos pavimentos e perspetivar intervenções de reabilitação. Neste sentido, foram modeladas diferentes intervenções, começando com a remoção das camadas existentes e a reconstrução da nova estrutura do pavimento (Figura 1). Esse processo possibilitou o cálculo da quantidade de cada material removido e sobreposto e, se disponível, o cálculo dos custos desses materiais.

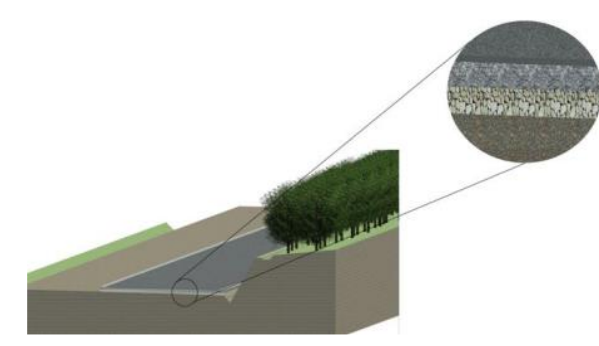

a)

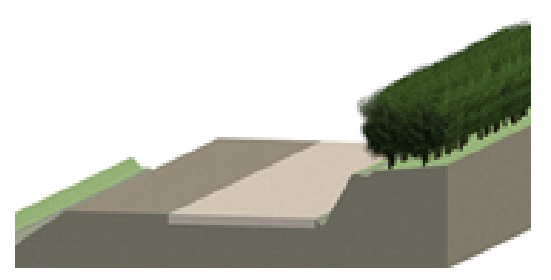

c)

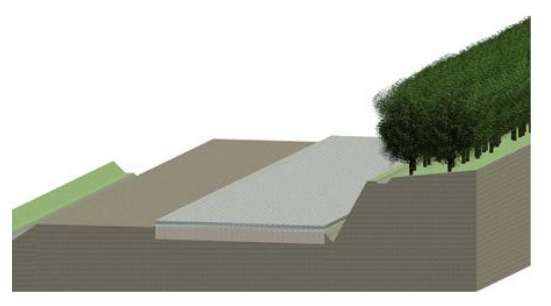

e)

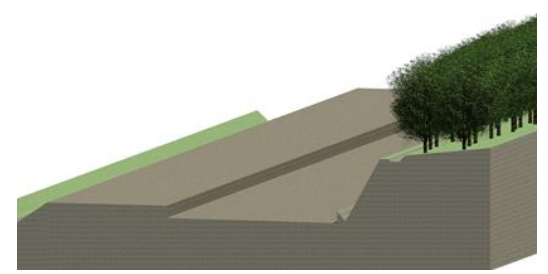

b)

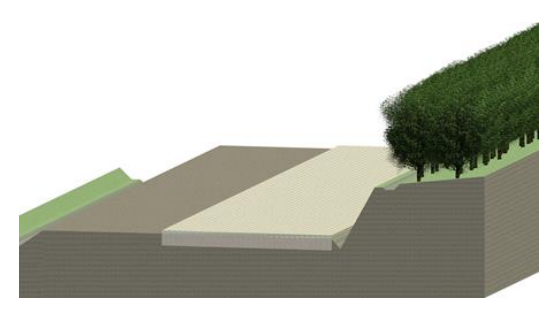

d)

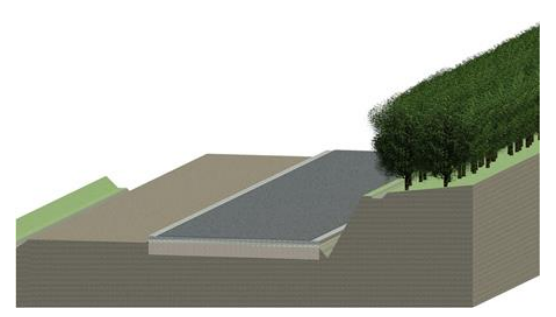

f)

Figura 1: Modelação das várias fases da intervenção de reabilitação empreendida na seção de controlo: a) Estrutura de pavimento existente; b) Remoção das camadas de pavimento; c) Camada de reforço de sub-base; d) Construção da camada de sub-base; e) Construção da camada de base; f) Nova estrutura de pavimento após intervenção.

Ainda no que se refere a pavimentos rodoviários, outra aplicação efetuada teve como objetivo modelar as características estruturais do pavimento medidas através de ensaios de carga com o Defletómetro de Impacto [4], tendo sido modelados dois parâmetros: i) a deflexão central (D1), que reflete as condições estruturais gerais do pavimento; e ii) a deflexão mais afastada (D7), localizada a 1,8m do centro da zona de carga, que reflete a rigidez da sub-base. A Figura 2 
mostra modelação em BIM do parâmetro D1 e a correspondente condição estrutural geral do pavimento refletida ao longo da seção de controlo [5].

$$
\text { deslocamento }\left(x 10^{-3} \mathrm{~mm}\right) \quad \begin{aligned}
& \square \\
& \square<0-500 \\
& \square<300 \\
& \\
& \square>500
\end{aligned}
$$

\section{Figura 2: Modelação BIM de D1 ao longo da secção de controlo.}

Usando os mesmos ensaios de carga não destrutivos e seguindo o procedimento para avaliação estrutural de pavimentos de aeroportos, o Número de Classificação de Pavimento (PCN) pode ser estimado para um pavimento aeroportuário, bem como sua evolução no tempo. Isso permite o controle de tráfego aéreo autorizados a usar o aeroporto e evita uma deterioração acelerada do pavimento [6]. A Figura 3 apresenta a modelação em BIM que incorpora a informação dos ensaios de PCN de uma pista aeroportuária [4] antes (1998 e 2000) e após intervenção de reabilitação (2002).

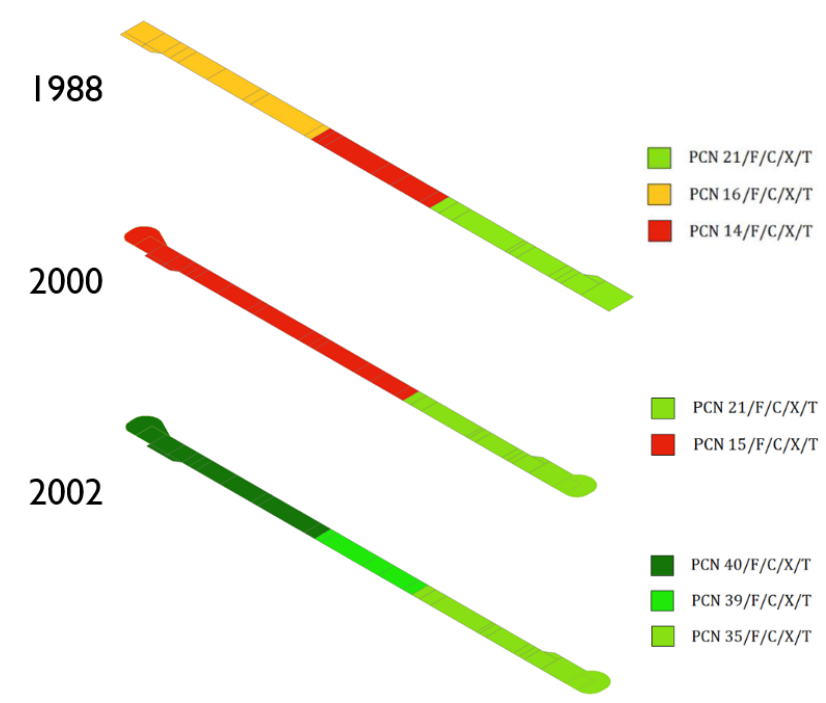

Figura 3: PCN de uma pista de aeroporto ao longo da sua vida útil, antes (1998 e 2000) e após intervenção de reabilitação (2002).

No caso de pavimentos aeroportuários, o coeficiente de atrito deve ser medido de forma sistemática, a fim de fornecer condições de segurança [5]. Essa avaliação é feita com equipamentos dedicados e, logo que se atinge um nível especificado definido pela Organização Internacional de Aviação Civil (OACI), é necessário planear campanhas de manutenção [6]. O modelo BIM pode também ser usado para armazenamento de informações atualizadas sobre a condição do coeficiente de atrito em pistas aeroportuárias, conforme apresentado na Figura 4. 


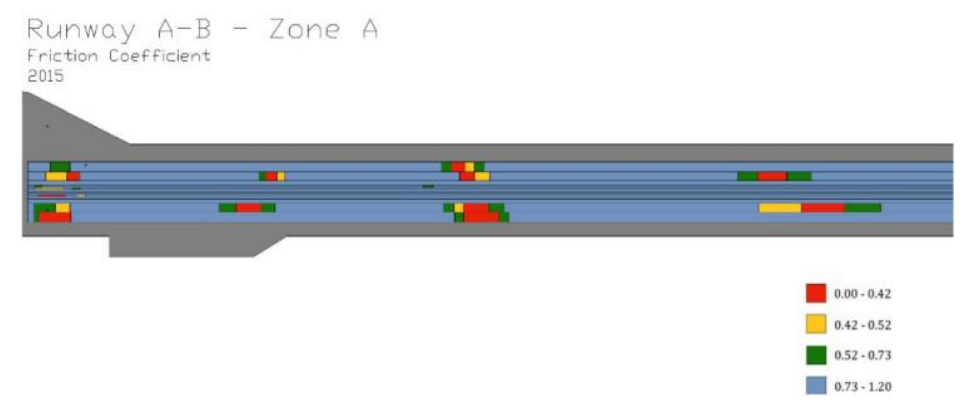

Figura 4: Coeficiente de fricção ao longo de pista aeroportuária.

Se atualizado regularmente, a modelação das características estruturais de pavimentos usando o BIM fornece um indicador visual da evolução das condições do pavimento ao longo do tempo, permitindo um melhor planeamento da sua gestão e manutenção (Figura 5).
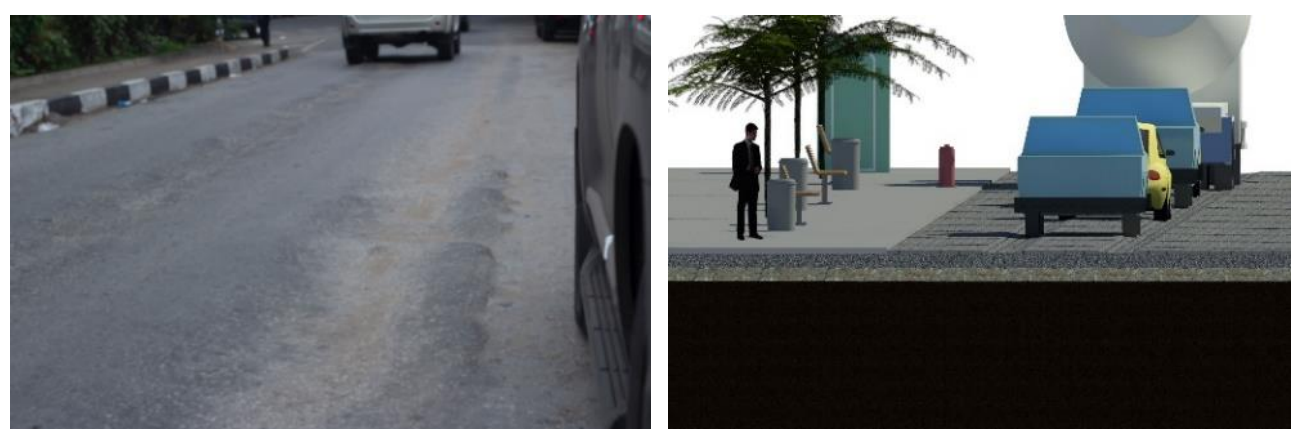

Figura 5: Pavimento urbano: a) resultados da inspeção visual (fotografia); b) resultados da inspeção visual (modelo BIM).

\section{BIM na gestão da via-férrea}

No caso de infraestruturas ferroviárias são apresentados dois casos de estudo: i) um durante a construção de uma nova linha e; ii) o outro antes e depois da renovação total de uma linha existente. O primeiro caso de estudo visa refletir o controle de qualidade durante a construção de uma nova linha. A modelação em BIM permite controlar a qualidade do trabalho durante a construção e a sua evolução ao longo do tempo. Neste caso foi estudada uma zona de transição (Figura 6), que representa a seção mais sensível da ferrovia à ocorrência de assentamentos ao longo do tempo [7].

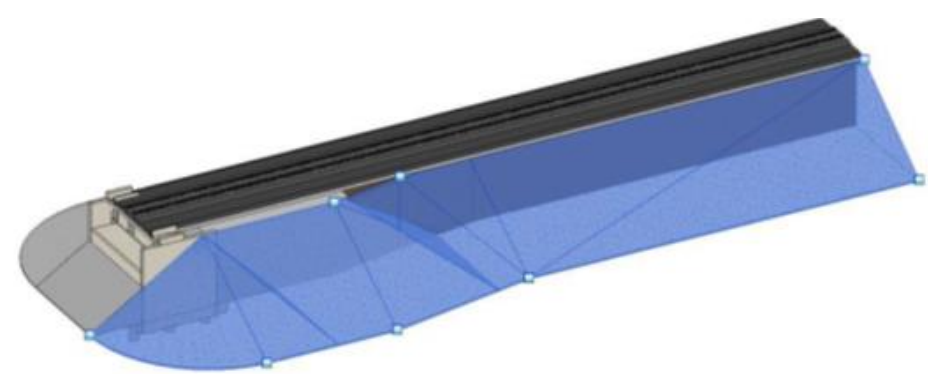

Figura 6: Modelo BIM de zona de transição da via-férrea. 
Na Figura 7 concretiza-se, em modelo BIM, a evolução dos assentamentos na camada de balastro durante os primeiros três anos após a abertura ao serviço e circulação de comboios da nova linha estudada. A informação obtida é clara e objetiva permitindo verificar a evolução registada no tempo, que, conforme se pode concluir foi diferente no lado esquerdo e no lado direito da via férrea.

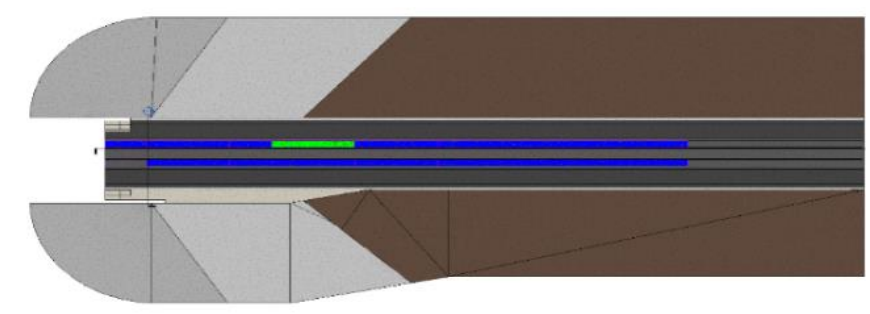

a) 13 meses após abertura da linha ao tráfego de comboios

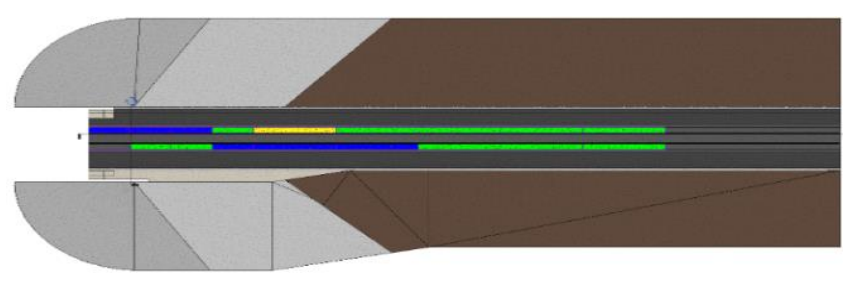

b) 24 meses após abertura da linha ao tráfego de comboios

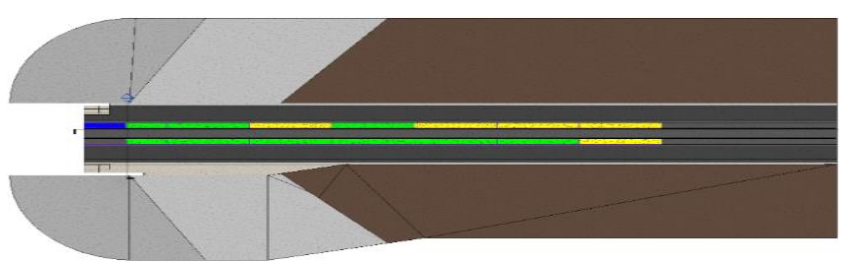

c) 36 meses após abertura da linha ao tráfego de comboios

$$
\text { deslocamento (mm) } \begin{gathered}
\text { oa } 10 \\
\text { 10 a } 12 \\
\text { 12 a } 14
\end{gathered}
$$

Figura 7: Modelação BIM de assentamentos na camada de balastro nos primeiros três anos de serviço.

O controle de qualidade, neste caso, deve ser muito rigoroso atendendo ao risco de descarrilamentos, devendo levar em consideração o efeito dinâmico dos comboios em circulação, dado que a rigidez da via pode mudar de forma muito significativa ao longo de vários trechos (por exemplo em zonas entre obras de arte e via normal ou entre via normal e zona de predominância de aterros). Torna-se, pois, importante em cada infraestrutura de transporte, ter uma referência "instante zero" para melhor acompanhamento do comportamento da resposta estrutural ao longo do tempo. O segundo caso de estudo considerado foi desenvolvido para acompanhar a qualidade da geometria da via numa linha existente e sua 
evolução quando foi empreendida uma renovação/reabilitação total [8][9]. A via férrea antes e depois da renovação foi modelada, tendo sido o seu nivelamento longitudinal representado no modelo BIM em escala de cores (Figura 8).

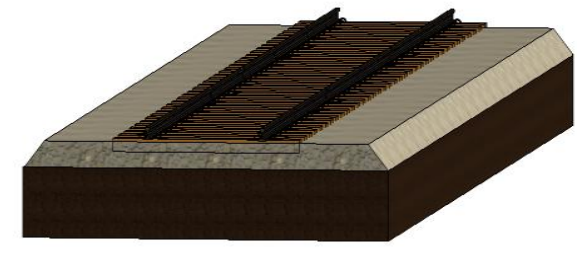

a)

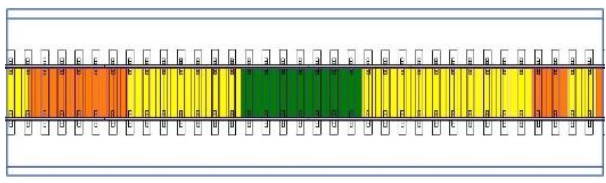

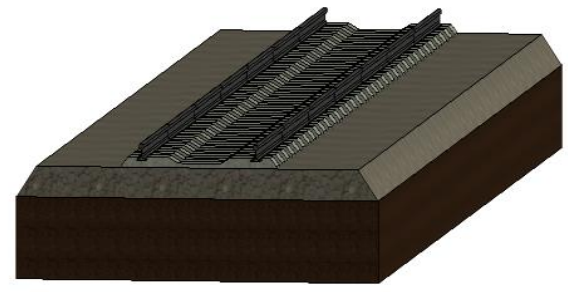

b)
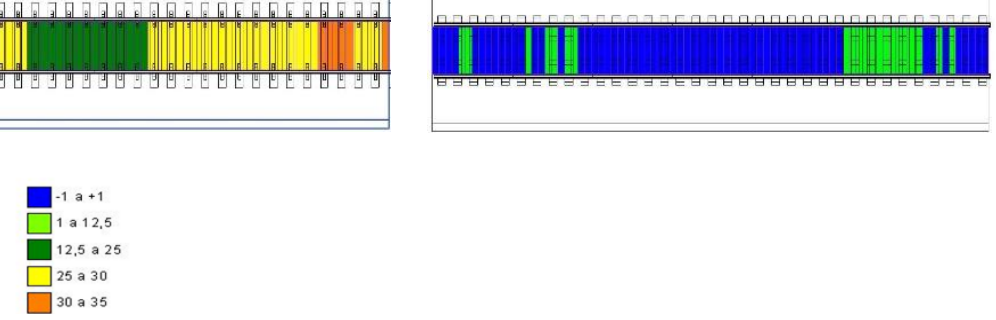

Deslocamento ( $\mathrm{mm})$

Figura 8: Modelo BIM da via e nivelamento longitudinal antes e depois de uma intervenção (renovação/reabilitação) total de uma linha ferroviária existente: a) Via férrea antes da intervenção; b) via férrea após intervenção.

Pode-se observar que a perceção visual da condição da bitola da via pode ser seguida neste modelo. É importante desenvolver ferramentas que permitam a importação automática de dados de medição para o modelo. Além disso, com base na modelação BIM, tanto de pavimentos (rodoviários e aeroportuários) quanto de via férrea, é possível estimar volumes e custos dos materiais necessários para intervenções de renovação / reabilitação, permitindo a escolha / seleção de soluções cada vez mais económicas e sustentáveis [10].

\section{Considerações finais}

Em todo o mundo as novas tecnologias transformam a maneira como as pessoas criam produtos, edifícios, infraestrutura e muito mais. As tendências tecnológicas atuais têm vindo a mudar a maneira como os profissionais do setor da Arquitetura, Engenharia, Construção e Operação (AECO) planeiam, projetam, constroem e mantêm o ambiente construído e os serviços públicos oferecidos à população. A tecnologia tem sempre transformado a maneira como as coisas são feitas e tem aberto oportunidades para determinar e moldar o nosso futuro. O BIM em projetos de infraestrutura de transportes traz vários benefícios acrescidos, tais como: i) melhor qualidade do projeto com maior adesão a normas; ii) melhor assertividade nos mapas de quantidades e orçamentos; iii) redução no tempo de aprovação e análise dos projetos; e iv) manutenção a partir do ativo virtual e real, possibilitando a ampliação e a manutenção preventiva a partir do modelo. 
Os casos de estudos apresentados no presente artigo ilustram as aptidões da aplicação do BIM em infraestruturas de transportes em diferentes fases do seu ciclo de vida. No entanto, há determinadas alterações que deverão ser empreendidas que permitirão a sua maior operacionalização para implementação generalizada a todos os tipos de infraestruturas de transportes. A modelação BIM da condição estrutural e funcional de pavimentos (rodoviários ou aeroportuários) ou de ferrovia é uma ferramenta útil ao longo do ciclo de vida que facilita a interpretação visual da evolução das suas condições de funcionamento no tempo. O processo de uso da escala de cores é útil, muito embora trabalhoso, precisando, em algumas circunstâncias, de ser adaptado aos valores dos parâmetros de monitorização representados. Como desenvolvimentos futuros, a importação automática de dados de monitorização para o modelo BIM pode melhorar significativamente o acompanhamento do desempenho da infraestrutura de transporte, permitindo a deteção e identificação de qualquer eventual comportamento de risco.

\section{Referências}

[1] C. M. Eastman, P. Teicholz, R. Sacks, and K. Liston, BIM handbook : a guide to building information modeling for owners, managers, designers, engineers, and contractors. Hoboken, N.J.: Wiley, 2008.

[2] S. Moreau "Level Of Development". https://bim42.com/2012/11/level-of-development/, 2012 (acedido a 15 de junho de 2020).

[3] S. Davidson, "What is BIM?" http://www.rics.org/uk/knowledge/glossary/bim-intro/, 2014 (acedido a 15 de junho de 2020).

[4] H. Manico "Flexible road pavements in Angola. Characterization and application of BIM methodologies," Dissertação de Mestrado, Faculdade de Ciência e Tecnologia, Universidade Nova de Lisboa (in portuguese), 2018.

[5] S. Fontul "Structural evaluation of flexible pavements using non-destructive tests" Tese de Dourtoramento, Universidade de Coimbra, Coimbra, Portugal, 2004.

[6] J. Lopes "Evaluation of airport infrastructures. Proposal for data integration in BIM," Dissertação de Mestrado, Faculdade de Ciência e Tecnologia, Universidade Nova de Lisboa (in portuguese), 2017.

[7] S. Carmali "BIM application to railway infrastructures. Quality control during construction," Dissertação de Mestrado, Faculdade de Ciência e Tecnologia, Universidade Nova de Lisboa (in portuguese), 2017.

[8] A. Pereirinha "Aplicação da metodologia do BIM na monitorização reabilitação e monitorização reabilitação de infraestruturas ferroviárias," Dissertação de Mestrado, Faculdade de Ciência e Tecnologia, Universidade Nova de Lisboa (in Portuguese), 2018.

[9] S. Fontul, A. Paixão, M. Solla, L. Pajewski "Railway Track Condition Assessment at Network Level by Frequency Domain Analysis of GPR Data," Remote Sens. 2018, 10(4), 559, doi: 10.3390/rs10040559, 2018.

[10]S. Fontul, p. Couto, M.J. Falcão Silva "BIM applications to pavements and railways. Integration of numerical parameters," CIAC2019 - Conferência sobre Inovação em Automação na Construção, Leiria, Portugal, 2019 